\title{
Desenvolvimento vegetativo em diferentes hastes da planta de mandioca em função da época de plantio
}

\author{
Vegetative development on different stems of cassava as a function of planting date
}

\author{
Lovane Klein Fagundes ${ }^{\mathrm{I}}$ Nereu Augusto Streck ${ }^{\mathrm{I}}{ }^{*}$ Sidnei José Lopes ${ }^{\mathrm{II}}$ Hamilton Telles da Rosa ${ }^{\mathrm{III}}$ \\ Lidiane Walter ${ }^{\mathrm{III}}$ Alencar Junior Zanon"III
}

\section{RESUMO}

Estudos sobre o desenvolvimento vegetativo da mandioca cultivada nas condições subtropicais do Brasil são escassos. O objetivo deste trabalho foi determinar o filocrono e o número final de folhas na haste principal e nas ramificações simpodiais de uma variedade de mandioca em diferentes épocas de plantio, em condições de clima subtropical. Um experimento de campo foi conduzido em Santa Maria, Rio Grande do Sul (RS), com quatro datas de plantio: 26/09/2006, 18/10/2006, 08/11/2006 e 28/11/2006. A variedade usada foi a FEPAGRO RS13, com caule do tipo simpodial tricotômico, no delineamento experimental inteiramente casualizado, com quatro tratamentos (datas de plantio) e quatro parcelas de 4,0 x 9,0m, com cinco fileiras e espaçamento $0,80 \times 0,80 \mathrm{~m}$. Em seis plantas por parcela selecionadas aleatoriamente, foi feita a contagem semanal do número de folhas visíveis (NF) e do número final de folhas (NFF) da haste principal (HP) e das ramificações simpodiais de primeira (RS1) e de segunda (RS2) ordem. $O$ filocrono $\left({ }^{\circ} \mathrm{C}\right.$ dia folha $\left.{ }^{-1}\right)$ foi estimado para $H P, R S 1$ e RS2 pelo inverso do coeficiente angular da regressão linear entre NF e soma térmica acumulada, considerando a temperatura base de $14^{\circ} \mathrm{C}$. O filocrono aumentou na seqüencia $H P<R S 1<R S 2$, e o NFF teve variação inversa $H P>R S 1>R S 2$. Ambos filocrono e NFF variaram com a época de plantio, sendo o fotoperíodo uma possível causa da variação. Dentro das ramificações simpodiais RS1 e RS2, o filocrono e o NFF não são diferentes entre as hastes.

Palavras-chave: filocrono, número de folhas, aparecimento de folhas, graus-dia, Manihot esculenta.

\section{ABSTRACT}

Studies on the vegetative development of cassava grown in subtropical conditions of Brazil are scarce. The objective of this study was to determine the phyllochron and the final leaf number on the main stem and on sympodial branches of a cassava variety grown in different planting dates in a subtropical climate. A field experiment was conducted in Santa Maria, RS, with four planting dates: 09/26/2006, 10/18/ 2006, 11/08/2006 and 11/28/2006. The variety FEPAGRO RS13, with tricotomic growth habit, was used in a complete randomized design with four treatments (planting dates) and four $4.0 \times 9.0 \mathrm{~m}$ plots with five rows and $0.8 \times 0.8 \mathrm{~m}$ spacing in each planting date. On the main stem (MS) and on the first order (BR1) and second order (BR2) sympodial branches of six plants per plot randomly selected, the number of visible leaves (NL) on a weekly basis, and the final leaf number (FLN) were measured. The phyllochron on MS, BR1 and BR2 was estimated by the inverse of the slope of the linear regression of $N L$ against accumulated thermal time, assuming a base temperature of $14^{\circ} \mathrm{C}$. Phyllochron increased in the sequence $M S<B R 1<B R 2$ and the FLN showed an inverse trend $M S>B R 1>B R 2$. Both phyllochron and FLN varied according to planting date, with photoperiod being a possible cause of such variation. Within BR1 and BR2 sympodial branches, phyllochron and FLN were not different among stems.

Key words: phyllochron, leaf number, leaf appearance, degreedays, Manihot esculenta.

\section{INTRODUÇÃO}

A mandioca (Manihot esculenta L. Crantz) é uma planta nativa do Brasil e foi extensamente disseminada pelos portugueses durante os séculos XVI e XVII para áreas tropicais e subtropicais da África, da Ásia e do Caribe. É base de múltiplos produtos, como

'Programa de Pós-graduação em Agronomia, Centro de Ciências Rurais (CCR), Universidade Federal de Santa Maria (UFSM), Santa Maria, RS, Brasil..

IIDepartamento de Fitotecnia, CCR, UFSM, Avenida Roraima, 1000, 97105-900, Santa Maria, RS, Brasil. E-mail: nstreck1@smail.ufsm.br. *Autor para correspondência.

II'Curso de Agronomia, CCR, UFSM, Santa Maria, RS, Brasil. 
farinha, alimento para animais, alimentos preparados, doces, álcool, amido para colar papéis e tecidos e também produtos biodegradáveis (FAO, 2001). A cultura da mandioca tem uma importância destacada nas pequenas propriedades rurais do Brasil, especialmente no Rio Grande do Sul, onde é usada para a alimentação humana e a animal.

Fatores ambientais como temperatura e fotoperíodo afetam o crescimento e o desenvolvimento da cultura de mandioca. A temperatura do ar afeta a brotação das manivas, a formação, o tamanho e a vida útil das folhas na planta, sendo o crescimento favorecido quando a temperatura média anual varia de $25^{\circ} \mathrm{C}$ a $29^{\circ} \mathrm{C}$, podendo tolerar temperaturas de $16^{\circ} \mathrm{C}$ a $38^{\circ} \mathrm{C}$ (ALVES, 2006). Em relação ao fotoperíodo, estudos indicam que a mandioca é uma planta de dia curto e alcança maiores produções de raízes com fotoperíodos entre 10 e 12 horas. Dias longos favorecem o crescimento da parte aérea e diminuem o crescimento das raízes de reserva, e dias curtos aumentam o crescimento das raízes de reserva e reduzem o crescimento da parte aérea (TERNES, 2002; ALVES, 2006).

É nas folhas que acontece a fotossíntese que transforma a energia solar radiante em energia química. A velocidade (ou taxa) de aparecimento das folhas e sua longevidade determinam o índice de área foliar verde que intercepta a radiação solar usada na fotossíntese. Assim, o número de folhas acumuladas (NF) e o número final de folhas (NFF) em cada haste do caule são importantes parâmetros do desenvolvimento vegetativo da mandioca. Alguns genótipos de mandioca apresentam o caule monopodial de porte ereto, enquanto outros genótipos têm caule simpodial de porte ramificado com duas (dicotômico), três (tricotômico) ou quatro (tetratômico) hastes, chamadas ramificações simpodiais (CARVALHO \& FUKUDA, 2006). O NF pode ser medido ou estimado. Um dos métodos de estimativa do NF mais usados é o que adota o conceito do filocrono, definido como o intervalo de tempo entre o aparecimento de duas folhas sucessivas na haste de uma planta (KLEPPER et al. 1982; WILHELM \& McMASTER, 1995; STRECK et al., 2005a,b; SCHONS et al., 2007). Uma unidade freqüentemente usada para representar tempo em plantas é a soma térmica, com unidade ${ }^{\circ} \mathrm{C} \mathrm{dia}^{-1}$, sendo que nesse caso o filocrono tem unidade ${ }^{\circ} \mathrm{C}$ dia folha ${ }^{-1}$ (XUE et al., 2004).

O Rio Grande do Sul (RS) apresenta, segundo a classificação climática de Köppen, clima Cfa (subtropical) e Cfb (temperado) (MORENO, 1961), estando localizado no limite de latitude para cultivo da mandioca, que é $30^{\circ}$ Norte e Sul (EMBRAPA, 2003). O ciclo de desenvolvimento de culturas tropicais, como a mandioca, em regiões extratropicais, apresenta peculiaridades, pois é interrompido pelas baixas temperaturas hibernais que fazem a planta de mandioca perder as folhas e permanecer em repouso até que a temperatura volte a aumentar na primavera. No Rio Grande do Sul, a planta de mandioca fica exposta a temperaturas e fotoperíodos variáveis durante a estação de crescimento. Por exemplo, em Santa Maria, RS, a variação da temperatura normal é de $13,5^{\circ} \mathrm{C}$ (média das temperaturas mínimas em outubro) a $30,4^{\circ} \mathrm{C}$ (média das temperaturas máximas em janeiro) e a variação do fotoperíodo é de 13,1 horas em outubro a 15,0 horas em dezembro.

Estudos de desenvolvimento da mandioca em resposta às variações dos elementos meteorológicos em regiões subtropicais do Brasil são escassos. Em um estudo recente, SHONS et al. (2007) relatam que o filocrono e o NFF de folhas na haste principal (HP) variaram com a época de plantio, enquanto que o número de folhas na haste principal, por ocasião do início de acumulação de amido nas raízes tuberosas, não variou em razão da época de plantio. Para genótipos de mandioca com caule simpodial, há formação de ramificações simpodiais de primeira e de segunda ordem durante a estação de crescimento.

Portanto, é pertinente estender o estudo realizado por SHONS et al. (2007) para ramificações simpodiais e comparar estes parâmetros do desenvolvimento vegetativo da mandioca entre a haste principal e as ramificações simpodiais, haja vista que a competição por fotoassimilados, água e nutrientes aumentou à medida que novas ramificações simpodiais foram surgindo na planta de mandioca. Além disso, dentro (em um mesmo nível) das ramificações simpodiais há competição entre hastes, e o efeito desta competição sobre o filocrono e o NFF ainda merece mais estudos, pois em modelos matemáticos é assumido que a velocidade de emissão de folhas é a mesma entre as hastes da ramificação simpodial (MATTHEWS \& HUNT, 1994; GRAY, 2000) e na prática, a campo, são verificadas diferenças de tamanho das hastes dentro de cada nível de ramificação. Assim, as lacunas nos estudos sobre o desenvolvimento vegetativo da mandioca nas condições subtropicais do Brasil constituíram a motivação para realizar este estudo.

O objetivo deste trabalho foi determinar o filocrono e o número final de folhas na haste principal e nas ramificações simpodiais de uma variedade de mandioca, em diferentes épocas de plantio, em condições de clima subtropical. 


\section{MATERIAL E MÉTODOS}

O experimento foi realizado durante o ano agrícola 2006/07 na área experimental do Departamento de Fitotecnia, da Universidade Federal de Santa Maria (UFSM) (latitude: $29^{\circ} 43^{\prime} \mathrm{S}$, longitude: $53^{\circ} 43^{\prime} \mathrm{W}$ e altitude: $95 \mathrm{~m})$. Santa Maria está localizada na região fisiográfica da Depressão Central do Rio Grande do Sul, uma das duas regiões preferenciais para o cultivo da mandioca no Estado (IBGE, 2005). O clima da região, segundo a classificação de Köppen, é Cfa, que significa subtropical úmido sem estação seca definida e com verões quentes (MORENO,1961). O solo do local é uma transição entre a Unidade de Mapeamento São Pedro (Argissolo Vermelho distrófico arênico) e a Unidade de Mapeamento Santa Maria (Argissolo BrunoAcinzentado alítico umbrico) (BRASIL, 1973; STRECK et al., 2008).

Foram realizadas quatro épocas de cultivo, com plantios em 26/09/2006, 18/10/2006, 08/11/2006 e 28/11/2006. As duas primeiras datas estão dentro do período recomendado para a cultura nessa região, que é setembro e outubro (CONCEIÇÃO, 1981), ou seja, após o repouso hibernal, quando as condições ambientais (temperatura) encontram-se favoráveis para a brotação das gemas. As duas últimas datas de plantio são consideradas tardias para essa região, pois a estação de crescimento fica curta para alcançar altos rendimentos. No entanto, em alguns anos em que as temperaturas são baixas na primavera, os agricultores locais plantam mandioca nessa época também. As manivas-semente eram provenientes de plantas usadas em experimentos realizados no ano agrícola 2005/06, na UFSM, da variedade FEPAGRO RS 13, da Fundação Estadual de Pesquisa Agropecuária do Rio Grande do Sul, sendo que é um genótipo adaptado e bastante usado no RS, com caule do tipo simpodial tricotômico (FEPAGRO, 2005).

Foi usado um delineamento experimental inteiramente casualizado, com amostragem na parcela. A área foi dividida em quatro parcelões, onde foram distribuídos os quatro tratamentos (datas de plantio). Cada parcelão foi dividido em quatro parcelas e cada parcela foi composta por uma área de 4,0 x 9,0m, com cinco fileiras de 9,0m de comprimento. Foi usada uma densidade 16.000 plantas ha-1 ${ }^{-1}$, no espaçamento de $0,8 \mathrm{x}$ $0,8 \mathrm{~m}$ entre plantas e entre fileiras. Cada parcela foi separada por uma faixa de circulação de $3,0 \mathrm{~m}$.

O solo foi preparado com duas gradagens, seguida de uma adubação NPK com a fórmula 05-20-20 na dose de $275 \mathrm{~kg} \mathrm{ha}^{-1}$, distribuída a lanço na área antes do plantio. O plantio foi realizado em cada época, com coveamento manual, usando-se manivas-semente de $0,20 \mathrm{~m}$ de comprimento e na profundidade de $0,10 \mathrm{~m}$.
Foram realizadas duas adubações de cobertura com nitrogênio, uma no início de acumulação de amido e a outra aos 40 dias após a primeira aplicação, na dose de $40 \mathrm{~kg} \mathrm{ha}^{-1}$ de uréia em cada aplicação. O controle de plantas daninhas foi feito manualmente, para evitar interferência desses fatores bióticos sobre o crescimento e desenvolvimento das plantas. Não foram realizadas irrigações, mas não houve sintomas aparentes de deficiência hídrica, indicando que a precipitação tenha suprimido a demanda hídrica da cultura durante a estação de crescimento.

Uma semana após a emergência das plantas, considerada quando $50 \%$ das mesmas estavam visíveis acima do nível do solo em cada parcela, foram selecionadas aleatoriamente as amostras, que foram constituídas por seis plantas em cada parcela, totalizando 24 plantas em cada parcelão (data de plantio). Nas plantas selecionadas, foram identificadas com arames coloridos a haste principal (HP) e as três hastes das ramificações simpodiais de primeira ordem (RS1) e de segunda ordem (RS2) (Figura 1). Nas hastes marcadas, foi realizada, semanalmente, a contagem do NF visíveis. Uma folha foi considerada visível quando as bordas de um dos lóbulos da folha não mais se tocavam (SCHONS et al., 2007). Nas hastes de cada ramificação também foi contado o NFF. Os valores das temperaturas mínima e máxima diárias do ar durante o período experimental foram medidos na Estação Climatológica Principal, pertencente ao $8^{\circ}$ Distrito de Meteorologia, do Instituto Nacional de Meteorologia, localizada a aproximadamente $150 \mathrm{~m}$ da área experimental.

A soma térmica diária (STd, ${ }^{\circ} \mathrm{C}$ dia) a partir da emergência das plantas foi calculada segundo ARNOLD(1960):

$\mathrm{STd}=(\mathrm{Tm}-\mathrm{Tb}) \cdot 1 \mathrm{dia}$

em que Tm é a temperatura média diária do ar, calculada pela média aritmética das temperaturas mínima e máxima do ar, e Tb é a temperatura base, considerada como $14^{\circ} \mathrm{C}$ para essa variedade de mandioca (SCHONS et al., 2007).

A soma térmica acumulada a partir da emergência (STa, ${ }^{\circ} \mathrm{C}$ dia) foi calculada por:

$\mathrm{STa}=\Sigma \mathrm{STd}$

(O filocrono, para a HP e para as hastes da RS1 e RS2, foi estimado como sendo o inverso do coeficiente angular da regressão linear simples entre NF e STa, a partir da emergência para a HP, e entre NF e STa a partir do aparecimento das ramificações simpodiais para RS1 e RS2)(KLEPPER et al., 1982; KIRBY, 1995; XUE et al., 2004). 


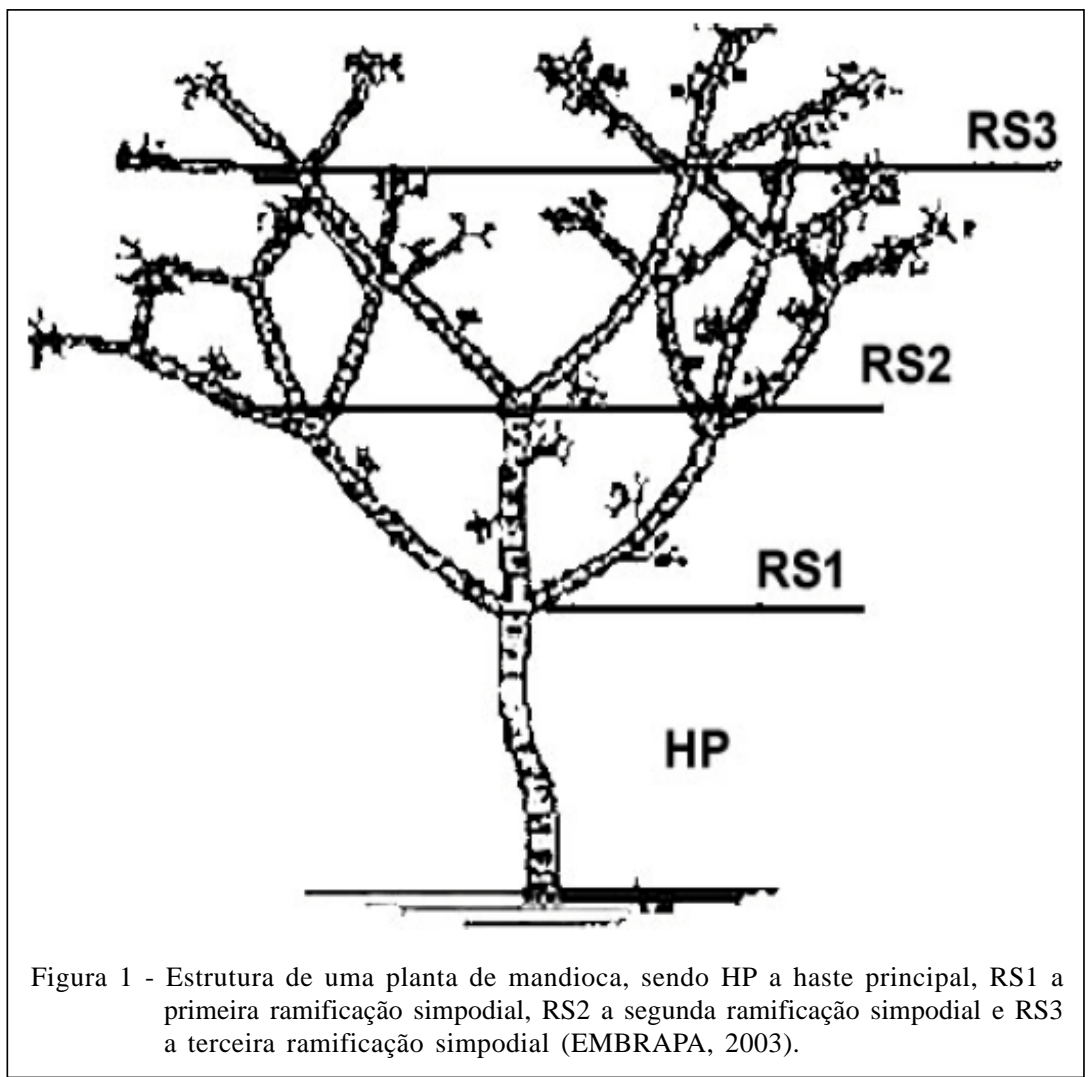

A análise estatística objetivou verificar diferenças (i) de filocrono e NFF de uma haste entre as ramificações (HP, RS1, RS2) e (ii) entre as hastes dentro um mesmo nível de ramificação simpodial (RS1 e RS2) nas plantas de mandioca, nas diferentes épocas de plantio. Para alcançar (i), foi considerado um bifatorial (quatro épocas $\mathrm{x}$ três hastes). Para alcançar (ii), foi considerado um fatorial hierárquico para as hastes de um mesmo nível de ramificação. As médias obtidas da análise bifatorial foram comparadas por meio do teste de Tukey, e as médias obtidas pelo fatorial hierárquico foram comparadas pelo teste $\mathrm{F}$, ambos a $5 \%$ de probabilidade de erro.

\section{RESULTADOS E DISCUSSÃO}

A temperatura média do ar durante o período de emissão de folhas na $\mathrm{HP}$ foi de $22,7^{\circ} \mathrm{C}, 23,9^{\circ} \mathrm{C}, 25,3^{\circ} \mathrm{C}$ e $25,6^{\circ} \mathrm{C}$, durante a emissão de folhas na RS1 foi de $25,6^{\circ} \mathrm{C}, 25,4^{\circ} \mathrm{C}, 25,0^{\circ} \mathrm{C}$ e $25,1^{\circ} \mathrm{C}$ e durante a emissão na RS2 foi de $25,1^{\circ} \mathrm{C}, 25,1^{\circ} \mathrm{C}, 22,9^{\circ} \mathrm{C}$ e $22,7^{\circ} \mathrm{C}$ nas datas de plantio 26/09/06, 18/10/06, 08/11/06 e 28/11/06, respectivamente. Já o fotoperíodo médio durante esses períodos foi 14,5, 14,9, 14,9 e 14,9h para HP, 14,8, 14,6, 13,5 e 13,5h para RS1 e 13,5, 13,5, 12,4 e 12,3h para RS2 nas quatro épocas de cultivo, respectivamente. Essas diferentes condições térmicas e fotoperiódicas em que as plantas foram submetidas são importantes neste estudo, pois representariam uma faixa ampla de condições ambientais para mandioca cultivada em regiões subtropicais, resultando em diferenças nas fases de desenvolvimento. Os períodos de fase de emergência até início da ramificação RS1 (EM-RS1) foram de 65, 63, 44 e 45 dias, de RS1 até início da ramificação RS2 (RS1-RS2) foram de 62, 42, 40 e 40 dias e de RS2 até início da ramificação RS3 (RS2-RS3) foram de37, 43, 37 e 38 dias nas quatro épocas de cultivo, respectivamente.

O coeficiente de determinação $\left(r^{2}\right)$ da regressão linear entre NF e STa nas diferentes épocas e hastes foi sempre maior que 0,9 , indicando que a temperatura é o elemento meteorológico principal que determina a velocidade de emissão de folhas nessa variedade de mandioca. Indica, também, que a estimativa do filocrono pelo inverso do coeficiente angular (declividade) da regressão linear é um método apropriado.

Na análise de variância (ANOVA) considerando o esquema bifatorial, houve interação significativa para a combinação época $\mathrm{x}$ haste para 
ambas as variáveis (filocrono e NFF), indicando que a análise estatística para a comparação de médias das duas variáveis deve ser desdobrada dentro de cada fator (Tabela 1). Nessa ANOVA entre os fatores principais (época e haste), a maior soma de quadrados foi para o fator haste, indicando que ambas as variáveis (filocrono e NFF) são mais influenciadas pela posição na planta do que pela época de cultivo.

Entre as hastes, o filocrono foi sempre maior na RS2, nas quatro épocas de plantio. O filocrono foi menor na HP, diferindo do filocrono da RS1 nas duas últimas épocas (11/08 e 28/11) (Tabela 1). Comparando o filocrono entre as épocas de plantio, não houve diferença de filocrono entre as datas para HP, sendo que houve diferença entre as duas primeiras épocas (menor filocrono) e as duas últimas épocas (maior filocrono) para RS1 e houve diferença entre duas épocas para RS2 (maior valor na data 08/11/2006 e menor na data 18/10/2006).

Para a variável NFF entre as hastes, a HP teve maior NFF em todas as épocas, com exceção da primeira época de plantio (26/09/2006), quando NFF foi menor que a RS1 (Tabela 1). Já o menor NFF foi na RS2 em todas as épocas de cultivo. Entre as épocas, o NFF na HP foi maior na terceira época (plantio em 08/11/ 2006) e menor na primeira época (26/09/2006). O NFF na RS1 foi diferente para todas as épocas, sendo maior na primeira época (26/09/2006) e menor na terceira época (plantio em 08/11/2006). Na RS2 o NFF foi maior na primeira época, não diferindo da segunda época, e menor na terceira e na quarta época, que não diferiram entre si (Tabela 1).

A hipótese para explicar o aumento do filocrono, que ocorreu na seqüência $\mathrm{HP}<\mathrm{RS} 1<\mathrm{RS} 2$ (com exceção do plantio em 18/10/2006 em que RS1<HP), é que quanto mais apical for a ramificação maior deve ser a competição dentro da planta, já que nessa variedade as ramificações simpodiais são tricotômicas. Assim, a cada nova ramificação simpodial três novas hastes aparecem e necessitam de fotoassimilados, nutrientes e água para crescerem e se desenvolverem. É notável que o filocrono aumenta mais da RS1 para a RS2 que da HP para RS1 (Tabela 1), o que pode ser explicado pelo fato de que o número de hastes em crescimento na RS1 é de três, enquanto que o número de hastes em crescimento na RS2 é de 9 . Tal fato confirma a hipótese de que o aumento da competição dentro da planta de mandioca é responsável pelo aumento do filocrono (diminuição da velocidade de emissão de folhas) em ramificações simpodiais de ordens maiores.

A variação do NFF nas hastes em diferentes ramificações teve tendência inversa àquela do filocrono, ou seja, NFF variou na seqüência HP $>$ RS1 $>$ RS2 (com exceção do plantio em 26/09/06 em que RS1>HP), o que também pode ser explicado pela hipótese de aumento da competição por fotoassimilados, água e nutrientes em ramificações de ordens maiores. Assim, quanto maior o número de ordem das ramificações simpodiais, maior o filocrono (menor a velocidade de emissão de folhas) e menor o NFF, evidenciando sincronismo no desenvolvimento vegetativo entre as ramificações da planta de mandioca.

Entre as épocas de cultivo, houve variação no filocrono para RS1 e RS2, enquanto que o NFF variou com a época de cultivo em todas as ramificações (HP, RS1, RS2) (Tabela 1). Testou-se a hipótese de que o fotoperíodo possa ter contribuído para alterar esses dois parâmetros do desenvolvimento vegetativo, por meio da regressão linear simples entre as variáveis filocrono e NFF (y) e o fotoperíodo médio (x) durante a emissão de folhas na HP, RS1 e RS2. Os resultados dessa regressão foram $y=-2,4 x+50\left(r^{2}=0,89\right)$ para filocrono na RS1, $y=-3,2 x+62\left(r^{2}=0,50\right)$ para filocrono na RS2, $y=55,7 x-769\left(r^{2}=0,36\right)$ para NFF na HP, $y=12,2 x+134\left(r^{2}=0,72\right)$ para NFF na RS1 e $y=6,5 x-65$

Tabela 1 - Filocrono $\left({ }^{\circ} \mathrm{C}\right.$ dia folha $\left.{ }^{-1}\right)$ e número final de folhas (NFF, folhas) em função da posição na planta (HP= haste principal, RS1= Ramificação simpodial de primeira ordem, RS2= Ramificação simpodial de segunda ordem) e da data de plantio da mandioca variedade de mandioca "FEPAGRO RS13". Santa Maria, RS, 2006/07.

\begin{tabular}{|c|c|c|c|c|c|c|}
\hline \multirow{2}{*}{ Época de plantio } & \multicolumn{3}{|c|}{-----------------------Filocrono---------------------- } & \multicolumn{3}{|c|}{-------------------------NFF-----------------------' } \\
\hline & $\mathrm{HP}$ & RS1 & RS2 & $\mathrm{HP}$ & RS1 & RS2 \\
\hline $26 / 09 / 2006$ & $13,7 \mathrm{Ba}$ & $14,4 \mathrm{Bb}$ & $21,2 \mathrm{Ab}$ & $41,7 \mathrm{Bd}$ & $52,4 \mathrm{Aa}$ & $23,8 \mathrm{Ca}$ \\
\hline 18/10/2006 & $14,7 \mathrm{Ba}$ & $13,9 \mathrm{Bb}$ & 18,3 Ac & $48,4 \mathrm{Ac}$ & $38,5 \mathrm{Bb}$ & $21,9 \mathrm{Ca}$ \\
\hline $11 / 08 / 2006$ & 13,8 Ca & $17,8 \mathrm{Ba}$ & $25,5 \mathrm{Aa}$ & 72,6 Аa & 28,6 Bd & $14,7 \mathrm{Cb}$ \\
\hline 28/11/2006 & $14,1 \mathrm{Ca}$ & $16,6 \mathrm{Ba}$ & $21,5 \mathrm{Ab}$ & $60,7 \mathrm{Ab}$ & $33,6 \mathrm{Bc}$ & $16,0 \mathrm{Cb}$ \\
\hline $\mathrm{CV} \%$ & 14,38 & & & 9,66 & & \\
\hline
\end{tabular}

* Médias não seguidas pela mesma letra maiúscula na horizontal e minúscula na vertical diferem pelo teste de Tukey a 5\% de probabilidade de erro. 
$\left(r^{2}=0,95\right)$ para NFF na RS2. Esses resultados indicam que o fotoperíodo pode ser um elemento meteorológico que afeta a velocidade de emissão de folhas na RS1 e RS2 e o NFF na HP, RS1 e RS2.

Dias longos (acima de 12 horas) favoreceram o crescimento vegetativo da mandioca (TERNES, 2002; ALVES, 2006). Neste trabalho, o fato de o fotoperíodo ter sido sempre superior a 12 horas e a regressão linear ter sido positiva entre NFF e fotoperíodo médio para HP, RS1 e RS2 sinaliza a favor dessa hipótese. No entanto, mais estudos precisam ser realizados para confirmar tal hipótese, dando-se prioridade para experimentos em que o fotoperíodo seja controlado, pois no campo o fotoperíodo é variável em nível diário e, ao fazer-se a média do fotoperíodo durante a emissão de folhas, podem ser introduzidos erros compensatórios, que reduzem a certeza da influência desse elemento sobre o desenvolvimento vegetativo dessa variedade de mandioca.

$\mathrm{Na}$ ANOVA considerando um fatorial hierárquico, não foi detectado efeito significativo do fator haste em cada ramificação simpodial (RS1 e RS2) para ambas as variáveis filocrono e NFF. Esses resultados indicam que o desenvolvimento das três hastes dentro de cada ramificação simpodial (RS1 e RS2) não é diferente nessa variedade de mandioca. A implicação desses resultados é que, em modelos de simulação do desenvolvimento da mandioca, pode-se usar a mesma velocidade de emissão de folhas nas diferentes hastes da mesma ramificação simpodial, confirmando que essa pressuposição usada para genótipos em outros países (MATTHEWS \& HUNT, 1994; GRAY, 2000) se aplica também a genótipos brasileiros de mandioca.

\section{CONCLUSÕES}

O filocrono aumenta e o número final de folhas diminui à medida que aumentam as ramificações simpodiais na planta de mandioca. Dentro das ramificações simpodiais da mandioca, o filocrono e o número final de folhas não são diferentes ente as hastes. O filocrono e o número de folhas em mandioca variam com a época de plantio.

\section{AGRADECIMENTOS}

À Fundação de Amparo à Pesquisa do Estado do Rio Grande do Sul (FAPERGS), pela Bolsa de Iniciação Científica a H.T. Rosa, e ao Conselho Nacional de Desenvolvimento Científico e Tecnológico (CNPq), pelo auxílio financeiro a N.A. Streck (Bolsa de Produtividade em Pesquisa) e a L.C. Walter (Bolsa de Iniciação Científica - PIBIC/CNPq/UFSM).

\section{REFERÊNCIAS}

ALVES, A.A.C. Fisiologia da mandioca. In: EMBRAPA Mandioca e Fruticultura Tropical. Aspectos socioeconômicos e agronômicos da mandioca. Cruz das Almas, BA: EMBRAPA, 2006. Cap.7, p.138-169.

ARNOLD, C.Y. Maximum-minimum temperatures as a basis for computing heat units. Proceedings of the American Society for Horticultural Sciences, Boston, v.76, n.1, p.682-692, 1960.

BRASIL, Ministério da Agricultura. Divisão de Pesquisas Pedológicas. Levantamento de reconhecimento dos solos do Rio Grande do Sul. Recife: MA/DPP, 1973. 431p. (Boletim Técnico, 30).

CARVALHO, J.E.; FUKUDA, W.M.G. Estrutura da planta e morfologia. In: EMBRAPA Mandioca e Fruticultura Tropical. Aspectos socioeconômicos e agronômicos da mandioca. Cruz das Almas, BA: Embrapa Mandioca e Fruticultura Tropical, 2006. Cap.6, p.126-137.

CONCEIÇÃO, A.J da. A mandioca. São Paulo: Nobel, 1981. p.382.

EMBRAPA. Mandioca e fruticultura: cultura da mandioca. 2003. Acesso em: 4 maio. 2007. On line. Disponível em: <http://sistemasdeproducao.cnptia.embrapa.br/ FontesHTML/ Mandioca/mandioca_amapa/sementes.htm>.

FAO [Food and Agriculture Organization of the United Nations]. The global cassava development strategy and implementation plan. Roma, 2001. V.1, 70p.

FEPAGRO. Novas cultivares para o Rio Grande do Sul: mandioca. Taquarí, RS: FEPAGRO, 2005. 3p.

GRAY, V.M. A comparison of two approaches for modelling cassava (Maninhot esculenta Crantz.) crop growth. Annals of Botany, Oxford, v.85, p.77-90, 2000.

IBGE. Produção Agrícola Municipal 2005: culturas temporárias e permanentes. Rio de Janeiro, Brasil: IBGE, Ministério do Planejamento Orçamento e Gestão, 2005. V.32, p.1-101, 2005.

KIRBY, E.J. Factors affecting rate of leaf emergence in barley and wheat. Crop Science, Madison, v.35, n.1, p.11-19, 1995.

KLEPPER, B. et al. Quantitative characterization of vegetative development in small cereal grains. Agronomy Journal, Madison, v.74, p.798-792, 1982.

MATTHEWS, R.B.; HUNT, L.A. GUMCAS: a model describing the growth of cassava (Manihot esculenta L. Crantz). Field Crops Research, Amsterdam, v.36, p.69-84, 1994.

MORENO, J.A. Clima do Rio Grande do Sul. Porto Alegre: Secretaria de Agricultura, Diretoria de Terras e Colonização, Secção de Geografia, 1961. 43p. 
SCHONS, A. et al. Emissão de folhas e início da acumulação de amido em raízes de uma variedade de mandioca em função da época de plantio. Ciência Rural, Santa Maria, RS, v.37, n.6, p.1586-1592, 2007.

STRECK, N.A. et al. Estimativa do filocrono em cultivares de trigo de primavera. Revista Brasileira de Agrometeorologia, Santa Maria, v.13, n.3, p.423-429, 2005a.

STRECK, N.A. et al. Estimating leaf appearance rate and phyllochron in safflower. Ciência Rural, Santa Maria, v.35, n.6, p.1448-1450, 2005b.
STRECK, E.V. et al. Solos do Rio Grande do Sul. 2.ed. Porto Alegre: Emater/RS-ASCAR, 2008. 222p.

TERNES, M. Fisiologia da planta. In: CEREDA, M.P. Agricultura: tuberosas amiláceas latino americanas. São Paulo: Fundação Cargill, 2002. V.2, p.66-82.

WILHELM, W.W.; McMASTER,G.S. Importance of the phyllochron in studying development and growth in grasses. Crop Science, Madison, v.35, n.1, p.1-3, 1995.

XUE, Q. et al. Predicting leaf appearance in field-grown winter wheat: evaluating linear and non-linear models. Ecological Modeling, Amsterdam, v.175, p.261-270, 2004. 